Revue d'histoire de l'Amérique française

ZRS REVUE D.HISTOIRE DE L'AMÉRIQUE FRANÇAISE

\title{
La mission du Père Paul Le Jeune, s.j., sur la Côte-du-Sud, 1633-1634
}

\section{Adrien Caron}

Volume 17, numéro 3, décembre 1963

URI : https://id.erudit.org/iderudit/302290ar

DOI : https://doi.org/10.7202/302290ar

Aller au sommaire du numéro

\section{Éditeur(s)}

Institut d'histoire de l'Amérique française

\section{ISSN}

0035-2357 (imprimé)

1492-1383 (numérique)

Découvrir la revue

Citer cet article

Caron, A. (1963). La mission du Père Paul Le Jeune, s.j., sur la Côte-du-Sud, 1633-1634. Revue d'histoire de l'Amérique française, 17(3), 371-395.

https://doi.org/10.7202/302290ar d'utilisation que vous pouvez consulter en ligne.

https://apropos.erudit.org/fr/usagers/politique-dutilisation/ 


\section{LA MISSION DU PĖRE PAUL LE JEUNE, S.J. SUR LA COTE-DU-SUD *}

\subsection{4}

Ceux qui ont voulu rappeler les difficultés que nos premiers missionnaires ont éprouvées parmi les sauvages, n'ont pu faire mieux que de transcrire ce que le Père Paul Le Jeune, jésuite, en a écrit dans la Relation de 1634: il y décrit ce qu'il a tout récemment vécu, et ses descriptions sont comme devenues classiques dans l'histoire de l'Église canadienne.

C'est ainsi que nous en trouvons de larges citations dans l'Histoire de la paroisse de Sainte-Foy du chanoine Scott, quoique l'auteur, très précis d'ordinaire, fasse hiverner le Père Le Jeune, avec ses Montagnais, dans la région du lac Saint-Jean.

Sur l'indication de l'abbé J.-B. Michaud, missionnaire de Pont-Viau, nous avons voulu suivre le Père Le Jeune et ses Montagnais pas à pas, en nous guidant sur la Relation de 1634, et nous avons passé l'hiver avec lui quelque part dans le comté de Kamouraska. Avec cette randonnée missionnaire du Père Le Jeune, c'est du vivant même de Champlain que commence l'histoire religieuse de la Côte-du-Sud, de la rive droite du Saint-Laurent en aval de l'île d'Orléans. Comme le remarquait l'abbé Michaud, "les aspects géographiques ne sont pas toujours les plus clairs" dans la narration du missionnaire. Il est certain cependant que l'hivernement a lieu du côté sud du fleuve, à quelques "journées de canot" de l'île d'Orléans. Par compa-

* Nota: l'on connaît bien cette relation de 1633-1634 du Père Paul Le Jeune. L'on connaît moins, du moins avec exactitude, le trajet du missionnaire s'attachant à suivre en ses chasses d'hiver, une tribu errante de Montagnais. M. l'abbé Adrien Caron a tenté de localiser, dans la géographie d'aujourd'hui, ce trajet du Père Le Jeune. Nous croyons que cet essai peut intéresser les chercheurs. (N.D.L.R.) 
raison et élimination, on peut situer de façon assez précise plusieurs des lieux décrits. Nous le faisons par mode d'hypothèse, sauf ce que l'on pourrait dire avec une étude plus parfaite des endroits et une certaine connaissance des noms indigènes.

Relevons d'abord l'itinéraire parcouru, quitte à revenir ensuite sur les incidents divers du voyage.

\section{LES LIEUX}

\section{A. LE TRAJET SUR LE FLEUVE}

Le Père Le Jeune écrit:

Je m'embarquai donc dans leur chaloupes le 18 octobre; nous allames descendre au-delà de l'Ile d'Orléans dans une autre isle nommée par les Sauvages Caouahasciumagakhe. Je ne scay si la beauté du jour se respandait desses cette isle, mais je la trouvai fort agréable.

En lisant ces lignes on pense tout naturellement à la Grosse Isle. Elle a un mille et demi de long. Du côté de l'île d'Orléans elle présente un promontoire d'une centaine de pieds d'élévation; sur le versant opposé le terrain descend en pente douce vers le rivage. A mesure qu'on approche de l'île on voit se détacher de la rive une presqu'île qui présente de chaque côté des anses sablonneuses qui facilitent l'arrivée en canot. Toute boisée alors, la Grosse Isle ne devait pas manquer d'être agréable à voir, alors que la forêt est revêtue de ses plus belles couleurs d'automne.

Le lendemain, 19 octobre, l'on tente de partir dès le matin, mais la marée, contraire, fait échouer la chaloupe. On se reprend le soir, et à la faveur du clair de lune, avec un bon vent, l'on aborde à une autre ile nommee Caouapascounagate.

Nous n'avons aucun détail sur cette deuxième île, aussi en aval de l'île d'Orléans.

Le lendemain, écrit le Père Le Jeune, nous quittasmes cette île pour entrer dans une autre appelée Cachibariouachgate. Nous la pourrions nommer l'Isle aux Oies blanches, car j'en vis plus de mille en une bande. 
Le jour d'après (le 21 octobre), nous la voulasmes quitter, mais nous fusmes contraints pour le mauvais temps de relascher au bout de cette mesme isle. Elle est déserte comme tout le pays, c'est-à-dire qu'elle n'a d'habitans qu'en passant, ce peuple n'a pas de demeure assurée; elle est bordée de rochers si gros, si hauts, et si entrecoupez et peuplee neantmoins de Cèdres et de Pins si proprement qu'un peintre tiendrait à faveur d'en avoir la vue. Sortant de cette isle au gibier, nous navigâmes tout le jour et vinrent descendre sur la nuict dans une petite islette nommée Atisaouacanichetagoukhi, c'est-adire le lieu ou l'on trouve la teinture (Il s'agit note le Père, de racines rouges).

Tout nous porte à croire que cette île au gibier, où il y a à l'automne des oies blanches par milliers, est bien l'île aux Oies actuelle. Les rochers du rivage et les récifs qui les prolongent paraissent toujours assez hauts à un homme assis au niveau de l'eau; l'île a même, près de la rive nord-ouest, son Gros Cap, comparativement haut, cent pieds d'altitude.

Nous croyons de même que cette îlette où l'on sera obligé de rester plus d'une semaine, à cause du mauvais temps, est bien celle qui a donné son nom à la paroisse de l'Islet. A cause de la tempête qui sévit tous les jours, le Père Le Jeune et ses Montagnais y sont comme prisonniers du 22 au 30 octobre. Cependant, profitant de cette inaction forcée, quelques sauvages vont visiter de leurs amis à 5 ou 6 lieues. C'est donc que cette îlette est accolée à la rive, détachée mais tout près du rivage, comme ce devait être le cas à l'Islet avant la construction du quai.

On pourrait objecter les mots "nous navigasmes tout le jour". Les Sauvages ont bien pu caboter un certain temps dans les îles, en pêchant et en chassant, avant de se décider à traverser au sud. Et si la mer était grosse déjà, avec un vent de nord-est, on en a eu assez pour la journée quand on a abordé à cette minuscule îlette de 4 ou 5 arpents de long. De cet endroit, en suivant les petits cours d'eau poissonneux, on compte bien 5 ou 6 lieues pour aller au lac des Trois-Saumons, un lieu favori des sauvages, comme d'autres ailleurs dans les environs. 
Comme nous y reviendrons, le Père Le Jeune a, lui aussi, profité de ce séjour forcé en cette îlette, pour faire pénétrer dans l'esprit de ses compagnons les premières vérités chrétiennes, inaugurant ainsi, à proprement parler, une première mission sur la Côte-du-Sud. Il poursuit:

Le trentieme jour d'octobre nous sortimes de cette malheureuse islette et vinsmes aborder sur la nuit dans une autre isle qui porte un nom quasi aussi grand comme elle est, car elle n'a pas demi-lieue de tour, et voici comme nos sauvages disent qu'elle se nommait: Capacoucach techekhi chachagou a chiganikhi.

Nous saurons par la suite que l'on peut, à mer basse, sortir de cette île au grand nom en traversant une prairie qui la sépare du continent. C'est, avec sa dimension, un indice précieux pour essayer de l'identifier.

Elle nous paraît être ce que les cartes modernes appellent la Pointe de Saint-André; autrement dit cet Islet du Portage qui a donné son nom à une seigneurie. Le manuel de géologie Laverdière-Morin en donne une photographie comme exemple de digue naturelle ou tombolo.

L'île de Cacouna est aussi reliée par une prairie à la rive droite du Saint-Laurent, mais elle a bien quatre milles de tour, tandis que cet Islet du Portage n'a qu'un mille de tour, pas une demi-lieue, comme écrit le Père Le Jeune. Élévation dépassant 100 pieds d'altitude. Entre l'îlette malchanceuse et cette île au grand nom, si ce sont bien les endroits que nous croyons, la distance est d'environ cinquante milles. La question se pose si l'on peut aller en chaloupe d'un lieu à l'autre, en partant le matin et en arrivant même tard dans la nuit.

M. Edgar Turgeon, commis-quincaillier à Québec, passait jadis sa semaine de vacances en chaloupe sur le fleuve, avec un M. Everell, un compagnon de travail. Nous l'avons consulté à ce sujet. Il estime qu'en partant de l'Islet à sept heures du matin on peut faire 25 milles en une marée et atteindre la pointe de la rivière Ouelle vers une heure de l'après-midi. Repartant de là à la marée suivante vers 7.30 du soir, arriver vers une heure et 
demie ou deux heures à la Pointe de Saint-André. Excellent problème de computation des lunes et des marées pour les connaisseurs.

\section{Continuons:}

Cette île (au grand nom) n'est quasi qu'un grand rocher affreux; comme elle n'a pas d'eau douce nous fusmes contrains de boire des eaux de pluies fort sales que nous ramassions dans des Fontaines et sur des rochers.

Nous trouvasmes en ce lieu la cabane d'un sauvage que notre hôte (Mestigoit) cherchait nomme Ekhennabamate.

Les Montagnais tinrent conseil et décidèrent de passer au côté nord du Saint-Laurent. Mais ayant fait à peine trois lieues sur le grand fleuve ils rencontrerent quatre canots de gens qui leur dirent que la chasse n'était pas bonne dans le nord. Alors tous ensemble s'en revinrent au sud, dans l'île d'ou l'on était parti.

\section{B. CAMPEMENTS EN FORET À L'INTÉRIEUR DES TERRES}

Le douze novembre, continue le Père Le Jeune, nous commencames en fin d'entrer dans les terres, laissant nos chaloupes et nos canots dans l'île au grand nom, laquelle nous sortismes de mer basse traversant une prairie qui la sépare du continent.

Comme on le voit, les quatre canots des sauvages venus du nord avaient augmenté la flottille et le nombre de bouches aussi, On devra se séparer plus tard à cause de la rareté du gibier.

Ici commence pour les Montagnais, comme pour le missionnaire qui les accompagne, une vie d'un autre genre. Le Père Le Jeune suspend sa narration pour nous présenter un coup d'œil d'ensemble. Nous lui en sommes reconnaissants.

Nous avons fait dans ces grands bois, dit-il, depuis le 12 novembre 1633, que nous y entrasmes, jusqu'au 2 avril de cette annee 1634, que nous retournasmes aux rives du grand fleuve Saint-Laurent, vingttrois stations, tantôt dans des vallees profondes puis sur des montagnes fort relevées, quelques fois en plat pays et toujours dans la neige. 
On ne saurait comparer les distances parcourues en chaloupe sur l'eau et les distances parcourues en forêt dans la boue et la neige. Il y a dans le groupe des hommes, des femmes, de jeunes enfants, même un qui est né en cours de route. Et l'on porte tout le bagage, même des écorces de bouleau que l'on transporte, quand on décabane, d'un campement à l'autre. Le territoire que représentent ces 23 stations ne s'étend pas plus qu'à trois ou quatre paroisses actuelles.

Le 12 novembre l'on va s'installer près d'un torrent. Nous croyons trouver ce torrent dans la rivière Fouquette qui arrive au Saint-Laurent à une lieue au nord-est de la Pointe SaintAndré. A l'oposé, au sud-ouest de la Pointe Saint-André, la première rivière rencontrée est la rivière Kamouraska, à trois lieues; elle coule en une plaine basse. Tandis que la rivière Fouquette arrive au fleuve entre deux coteaux élevés où son cours, descendant de 25 pieds au mille, est très rapide. Son débit était bien plus considérable jadis et on pouvait certes l'appeler un torrent.

Le 20 novembre, ne trouvant ni castors ni porcs-épics, l'on change d'endroit et on fait une deuxième station. Le 28 novembre de nouveau l'on décabane. Huit jours, donc, en chacun de ces endroits. Le 3 décembre, toute la bande se déplace de nouveau; ce sera la quatrième station, au sujet de laquelle le Père Le Jeune écrit:

Nous plantasmes notre camp proche d'un grand fleuve large et rapide mais peu profond; ils le nomment Capititetchouetz. Il va se dégorger dans le Grand Fleuve quasi vis-a-vis Tadoussac.

Ce fleuve arrivant au Saint-Laurent un peu avant Tadoussac devrait être la rivière du Loup. On peut le traverser sans embarcation, même si on a de l'eau à la ceinture ou que l'on doive nager au milieu, il est peu profond, dit-on. Assez large cependant... l'eau est froide à cette saison, et il a bien trois quarts d'arpents de large en arrière de Saint-Alexandre. Le Père Le Jeune y revoit la Marne à Châlons, son pays natal; elle ne doit pas y être plus large et on l'appelle un fleuve. 
Le six decembre nous delogeasmes pour la cinquieme fois. Le vingt decembre, malgre la pluie, il faudra encore decabaner. Le vingt-quatre decembre nous decampasmes pour la septieme fois.

Plus aucune indication locale depuis la traversée du fleuve Capitetchouetz le 3 décembre. En conséquence il est impossible de localiser le site du premier Noël célébré au diocèse de SainteAnne-de-la-Pocatière. Solennité à tout point de vue des plus pauvres, comme nous verrons. Mais la troupe, une fois traversée la rivière du Loup, a continué vers le sud, peut-être en suivant les rivières Fourchue et Rocheuse. Nous pourrons nous orienter de nouveau en janvier 1634.

Le huitième campement a lieu le 30 décembre et le neuvième le 4 janvier 1634 .

Le seizieme jour du mesme mois, nous battismes la compagne et ne pouvant arriver au but que nous prétendions, nous ne fismes que gister dans une hostellerie que nous dressasmes à la hâte, et le lendemain nous poursuivions notre chemin, passans sur une montagne si haute, qu'encore nous ne montassions point jusqu'au sommet, qui nous paraissait orné d'horribles rochers, néantmoins le Sorcier me dit que si le ciel obscurcy d'un brouillard eust été serain, nous eussions, veue à mesme temps Kébec et Tadoussac, éloignez l'un l'autre de quarante lieues pour le moins.

Ces pauvres sauvages, que nous sommes portés à croire terre à terre, savaient donc apprécier les beaux panoramas de leur pays. Ils regrettent qu'un temps brumeux empêche le Père de les admirer lui aussi.

Le Père Le Jeune raconte ensuite les difficultés qu'ils éprouvèrent à la descente de la montagne.

Le vingtneufiesme jour nous achevasmes de descendre de cette montagne, portant notre maison sur la pente d'une autre ou nous allasmes.

Voila le terme de notre pelerinage; nous commencions dorénavant à tourner bride et a tirer vers l'isle ou nous avions laisse notre chaloupe. 
Nous vismes les sources de deux petits fleuves qui se vont rendre dans un fleuve aussi grand, au dire des Sauvages, que le fleuve Saint-Laurens, ils l'appellent Ouera Ouachticou.

Dans ce fleuve que l'on dit comparable au Saint-Laurent, il est difficile de ne pas voir le fleuve Saint-Jean. Le nom sauvage que lui donne le Père Le Jeune a de la similitude avec celui de la carte Taché (1885) qui l'appelle Wall-as-took. Or précisément sur les confins des deux versants, du Saint-Laurent d'un côté et du fleuve Saint-Jean de l'autre, se trouve la montagne Bleue aux pentes plutôt raides, d'une altitude de 2150 pieds.* Il paraît donc certain que ce sommet est la montagne au panorama immense dont parle le Père Le Jeune.

Quant à l'expression "de Kébec à Tadoussac" il ne faut pas y voir une de ces exagérations dont les Sauvages avaient l'habitude; mais la seule manière de dire alors possible, faute de lieux suffisamment connus entre ces deux points extrêmes.

Il faut se rappeler que presque 50 ans après, en 1670 , le notaire Rageot, de Québec, en détachant de la seigneurie des Aulnaies le fief Pollet, en sera encore à le borner en disant: "du côté de Québec" et "du côté de Tadoussac".

En février on se déplace quatre fois, les 5, 9, 14 et 15 de ce mois. On est plus stable ensuite puisque la troupe reste en place jusqu'au 6 de mars. Nouveau déménagement après une semaine, le 13 de mars; c'est le dix-huitième campement. Les indications locales font défaut. On se dirige vers le lieu du débarquement; peut-être l'a-t-on aperçu de loin du haut des montagnes, à environ 6 lieues.

Ce dix-huitième campement dure dix jours, du 13 au 23 mars. Le dix-neuf mars, fête de saint Joseph, et, comme tous les autres jours, bien péniblement, le Père Le Jeune a lu son bréviaire. Il se trouvait, à n'en presque pas douter, sur le territoire de la paroisse actuelle de Saint-Joseph-de-Kamouraska.

* Station géodésique "Parke". 
Le vingt-trois mars on repasse le fleuve Capitetchouets que l'on a passé le trois decembre (on lit ici Capitetchionez).

Sur la rive droite de la rivière du Loup, vis-à-vis la Pointe de Saint-André c'est, en effet, dans Saint-Joseph aujourd'hui. Le 30 mars il écrit:

Nous vinsmes cabaner sur un fort beau lac en ayant passe un plus petit en notre chemin.

Le premier avril nous quittames ce lac. Enfin j'arrivai sur les rives du Grand Fleuve et trois jours apres nous fismes notre vingt-troisieme station dans l'ile ou nous avions laisse notre chaloupe.

Un lac entre la rivière du Loup et le fleuve Saint-Laurent à Saint-André, ce n'est pas sur toutes les cartes. Une carte provinciale de 1954 met cependant un petit lac bleu aux sources de la rivière Fouquette, au nord-est de la station de Saint-André. Une carte militaire plus récente donne ce lac comme saisonnier. Il était certainement plus grand autrefois, et il ne faut pas oublier que le Père Le Jeune est passé à la fonte des neiges, le 30 mars.

\section{LE RETOUR À QUÉBEC}

L'on est revenu à l'île au grand nom, Islet du Portage, aujourd'hui Pointe de Saint-André, le 4 avril. Dès le lendemain le Père Le Jeune part pour Québec.

Le cinq avril l'on s'embarqua dans un petit canot pour tirer sur Kebec.

Le retour fut très pénible. A cette saison il y a encore des glaces sur le Saint-Laurent, glaces flottantes au large, glaces en champs immenses sur les battures du rivage. Gagner la terre est difficile, circuler en forêt presque impossible. On dirait que le Père, malade depuis plusieurs semaines, n'a pas attendu le gros de la bande avec la chaloupe, puisqu'il dit qu'il est venu en petit canot.

Avec le montant, donc, on atteint à la première marée la rivière Ouelle, puis à la marée suivante la Grande Pointe de 
Saint-Roch; le lendemain l'île aux Oies, et ensuite la Grosse Isle. Simples suppositions.

Chose certaine, le 7 avril, on touche à la pointe Argentenaye, maintenant Saint-François de l'île d'Orléans et le 9, jour de Pâques, le Père Le Jeune se trouve à la résidence des Jésuites près de la rivière Lairet à Québec.

Il lui restait à rédiger, jusque tard dans la nuit, les notes si intéressantes qu'il a laissées sur son voyage et à mettre en ordre la liste des mots et des expressions montagnaises dont le recueil pourra servir aux autres missionnaires.

L'abbé Scott écrit dans son Histoire de Sainte-Foy:

Le Père LeJeune a laissé, dans la Relation de 1634, sur les coutumes, les mœurs et les superstitions des Sauvages, une description approfondie et complète, que les historiens ont ensuite exploitée, souvent sans en indiquer la source.

Et, honneur étant donné à qui de droit, le curé de Sainte-Foy résume ainsi :

De la mi-novembre 1633 jusqu'en avril 1634 le Père $P$. Le Jeune suivit les chasseurs à travers les bois, franchissant avec eux montagnes et vallées, lacs et rivières couverts de glaces. Après avoir pris un morceau le matin, on marchait tout le jour, sac au dos, sans rien prendre jusqu'au campement du soir. Alors les hommes, à l'aide de leurs raquettes, enlevaient la neige sur un petit espace circulaire, dressaient au-dessus, en les attachant par le haut, de longues perches coupées par les femmes, auquelles incombaient les travaux les plus rudes, et les recouvraient d'écorces qu'ils trainaient avec eux.

$\mathrm{Au}$ sommet une ouverture pour la fumée; d'un côté une entrée fermée d'une peau d'our; sur le sol gelé et les parois de neige une tapisserie de branches de sapin: c'était la cabane où le vent et le froid pénétraient de toute part.

Après avoir allumé un grand feu, on jetait dans une chaudière remplie de neige fondue et revêtue d'une couche épaisse de saleté, des pièces de viande qu'on ne lavait jamais de peur d'en perdre la graisse. La 
terre tenait lieu de table et chacun s'assoupissait auprès de son ouragan, sorte de plat d'écorce, où les chiens, toujours nombreux, avaient souvent plus vite mis leur nez que les convives leurs doigts, seuls instruments à l'usage des Sauvages dans leurs repas. Il fallait ensuite dormir dans cette étroite cabane où un froid excessif succédait à une chaleur suffocante, où l'on était aveugle par la fumée, brûlé d'un côté et gelé de l'autre. Les hommes, les femmes, les enfants, dix-neuf en tout, s'étendaient pêle-mêle avec les chiens qui, changeant souvent de place et peu délicats, mettaient indistinctement les pattes sur le ventre ou le visage des dormeurs et allaient se coucher sur le dos du premier venu.

Le pauvre missionnaire qui n'avait pour matelas et pour couverture qu'une simple catalogne, n'était pas faché qu'un de ces animaux choisit pour couche ses pieds ou ses épaules, afin d'en tirer un peu de chaleur.

Cet hivernement héroïque du Père Le Jeune parmi les Montagnais ne devait pas seulement aider les missionnaires à parler les langues sauvages et procurer à la postérité des récits intéressants. Il avait été, au prix de grands sacrifices, une expérience qui devait être décisive dans l'orientation des missions du Canada. Nous y reviendrons plus loin.

\section{LE MISSIONNAIRE}

C'est un vrai drame missionnaire que nous raconte tout bonnement le Père Paul Le Jeune dans le voyage de 1633-1634. Nous en connaissons le théâtre ou les lieux, présentons maintenant les acteurs ou les personnages.

\section{A. LES PERSONNAGES}

Le héros du drame est le narrateur lui-même. Aller hiverner dans les huttes rudimentaires des sauvages était héroïque, certes. Mais une fois sa décision prise, les adversités et les souffrances quotidiennes paraissent devenir choses ordinaires pour notre missionnaire. Il les dit simplement, et, sincèrement, il reconnaît tous ses insuccès. 
A côté du Père Le Jeune est celui qu'il nomme l'Apostat ou le Renégat. Son vrai nom est Pastedechouan. C'est un converti que les Pères Récollets avaient jadis amené tout jeune en France. Encore enfant il avait été baptisé solennellement à Angers. Conséquemment il s'exprime assez bien en français, mais à son retour il a été plutôt mal vu de ses compatriotes. On ridiculise cet individu moitié algonquin moitié français, qui aime la vie sédentaire et s'égare dans la forêt. La perfection pour les sauvages est d'être vagabond, vivre au jour le jour et parfois aussi crever de faim.

Une fois retourné parmi les siens, voulant sans doute se montrer des leurs, l'apostat avait abandonné toute pratique religieuse. Il ne gagna pas pour cela l'estime de ses compatriotes. Il était d'ailleurs, nous le verrons, mauvais chasseur, et cela eut suffi à lui ôter toute autorité en fait d'apostolat... Au cours de l'été de 1633, l'apostat était revenu de Tadoussac à Québec, pressé par la faim. Ayant entendu parler de lui, le Père Le Jeune voulait le rescaper, le ramener aux pratiques chrétiennes. Pendant leur séjour à Tadoussac, de 1629 à 1632, les Anglais avaient donné aux sauvages des boissons enivrantes, et l'apostat s'était laissé aller à l'ivrognerie. Il aimait toujours les Pères; il le dira même lorsqu'il sera en boisson. Volontiers il leur rendra service et c'est avec lui que le Père Le Jeune amorce le marché qu'il conclut avec les Montagnais pour qu'ils l'acceptent avec eux durant un hiver. Quant à aider le Père dans son apostolat, c'est autre chose; il ne se sent pas assez bien vu de ses compatriotes. Il consentira à traduire en langue sauvage, pour le Père, des prières et un peu de doctrine. Il ne voudra pas cependant répéter pour le missionnaire ce que celui-ci a essayé de dire aux sauvages dans son vocabulaire indigène rudimentaire.

Le cas de l'apostat était un peu celui d'un distingué catholique japonais, diplômé en Europe, qui avait donné en Amérique du Sud des conférences en langue espagnole et portugaise. Le missionnaire des Japonais de Lima l'invita à venir y parler à ses compatriotes. Il s'y rendit et leur fit un beau discours patriotique, se disant à peine catholique devant eux. 
Un jour l'apostat avoue au Père qu'il manquait totalement de volonté. Avec les Anglais, dit-il, je disais comme les Anglais, avec les Pères je dis comme eux, avec les sauvages je fais de même; plût à Dieu que je fusse mort alors que j'étais en France. De fait, il était totalement dans la dépendance de son frère Carigonan ou le Sorcier. Les missionnaires connaissent tous de ces gens qui voudraient bien mais ne peuvent avancer sans le consentement de quelque membre de leur famille.

Le Sorcier ou le Magicien, de son vrai nom Carigonan, fut aussi de l'expédition, en dépit de la promesse que Mestigoit, le chef de la bande, avait faite au Père de ne pas le prendre avec eux.

Cette présence du Sorcier fut fort gênante pour le missionnaire et contrecarra plus d'une fois son action. Tout de même, dans les moments où le Sorcier n'était pas là, le Père réussit à parler de religion aux sauvages, surtout avec Mestigoit qui paraissait bien disposé. Et même ses discussions avec le Sorcier ne durent pas être inutiles.

Nous avons nommé Mestigoit. C'est, dit le Père Le Jeune, un homme d'environ 35 ans, excellent chasseur, d'un bon naturel. Lorsque le Père s'embarqua avec lui, Champlain, qui s'intéressait à cette expédition missionnaire, lui recommanda de prendre bien soin du Père. S'il le faut, répondit Mestigoit, je mourrai avec lui, autrement on ne me reverra plus dans ce pays-ci. Le Père Le Jeune l'appelle d'ordinaire son hoste. Mestigoit s'intéressait un peu à tout, il questionnait même. Des choses naturelles le Père essayait de passer aux notions surnaturelles.

Il y a encore un autre sauvage dont on connaît le nom, à savoir Ekhennabamate, de l'île au grand nom. Et aussi l'Iroquois, peut-être un esclave de guerre pris tout jeune, ou un bâtard né d'un père Iroquois. Il paraît un sauvage de seconde zone, puisqu'on ne l'amène pas à la chasse, on le laisse avec les femmes ... faire les travaux pénibles.

La bande compte en tout au départ une vingtaine de sauvages, hommes, femmes et enfants. Le Père est le seul Français 
avec eux. On sera à un moment donné 45 personnes ensemble, avec le groupe de l'île au grand nom et ceux des quatre canots venus du nord. Ces derniers, à cause de la rareté du gibier, prirent ensuite leur côté. À Noël, on était une trentaine en tout.

\section{B. À LA MERCI DE LA CRAINTE}

Pendant la semaine où les tempêtes le retinrent dans l'îlette malheureuse, c'est-à-dire du 22 au 30 octobre 1633, le Père Le Jeune put constater que quelques craintes superstitieuses asservissaient ces pauvres sauvages, les femmes surtout.

Le Père Morice, o.m.i., raconte des scènes analogues chez les Dénés de l'Ouest. A un moment donné quelqu'un crie: Dénégéré (l'homme ennemi, inimicus homo) c'est-à dire le diable, et, précipitamment, tous se dispersent à l'épouvante.

À l'îlette, alors que presque tous les chasseurs étaient partis depuis quelques jours, il arriva quelque chose de semblable, à ce que raconte le Père Le Jeune:

La nuit, dit-il, une femme étant sortie s'en revint toute effarée criant, qu'elle avait ouy le Manitou ou le Diable. Voila l'alarme dans notre camp; tout le monde, remply de peur, garde un profond silence. Je demande d'ou provient cette épouvante, car je n'avais pas entendu ce qu'avait dit cette femme. Ecatitou, Eca titou, dit-on, Manitou. Tais-toi, taistoi, c'est le Diable.

Je me mis à rire et me levant en pied je sors de la cabane, et pour les assurer j'appelle en leur langue: Manitou, Manitou, criant tout haut que je ne craignais pas et qu'il n'oserait venir ou j'estois. Puis, ayant fait quelques tours dans notre Islette je rentrai et leur dit: Ne craignez point, le Diable ne vous fera aucun mal tant que vous serez avec moi. Il craint ceux qui croient en Dieu, si vous y croyez, il s'enfuira de vous.

Les femmes jetaient des morceaux d'anguille au feu. Le Père demanda pourquoi. C'est pour donner à manger au Manitou, répondirent-elles. À son retour de la chasse Mestogoit remercia le Père d'avoir rassuré les femmes. Il n'aurait pu en faire autant, car il avait lui-même autant peur qu'elles du Manitou, et l'avouait. 
Note: Manitou paraît signifier un esprit, bon ou mauvais. Les sauvages connaissant fort peu les bons esprits, Dieu et les Anges, et craignant grandement les mauvais, il s'ensuit qu'en pratique le mot Manitou est ici synonyme de Diable. De même en grec daimon veut dire un esprit, bon ou mauvais. Mais les Grees ne connaissaient pas plus que les sauvages les bons esprits, et daimon était plutôt un mauvais esprit, un diable. C'est pourquoi les Chrétiens ont adopté un autre mot angellos, pour désigner les bons esprits.

\section{PREMIERS ENSEIGNEMENTS}

Pour donner aux sauvages les premières notions sur Dieu et la religion, le Père Le Jeune pensa que rien n'était mieux que quelques prières faciles en leur langue. C'était pratique de leur donner, en même temps que les premières vérités de la foi, un moyen concret de vivre de cette foi, en orientant leur vie et leurs actions vers Dieu.

Je dressay, dit-il, quelques prières en leur langue, avec l'aide de l'Apostat. Comme le Sorcier n'était pas encore venu, je les récitais le matin et avant les repas, eux-mêmes m'en faisant souvenir et prenant plaisir à les ouyr prononcer.

Si ce miserable Magicien n'était pas venu avec nous, ces Barbares auraient pris grand plaisir à nous écouter. Mon hoste me faisait mille questions, me demandant pourquoi nous mourrions, ou allaient nos âmes, si la nuit était universelle pour tout le monde et choses semblables, se montrant tres attentif à mes reponses.

Pendant le séjour dans l'île au grand nom, les sauvages, pour s'amuser * de sa mauvaise prononciation, prièrent le Père Le Jeune de leur parler en langue sauvage. Il acquiesça volontiers, pour entrer en contact avec eux. On lui demandait si la lune était aussi haute que les étoiles, où allait le soleil quand il nous quittait: Comment peux-tu en savoir plus long que nous sur tout cela, lui demandaient les sauvages? Tirant sa montre, le missionnaire leur expliqua les fuseaux horaires. Quand je saurai votre langue, ajouta-t-il, je vous expliquerai beaucoup de choses.

* Même chose pour le Père Turquetil. Lionel Groulx, ptre, Le Canada français missionnaire, $\mathbf{5 1}$. 
Tu as de l'esprit, tu le sauras bientôt, lui dirent-ils.

Ils se sont trompez, affirme le Père Le Jeune.

A l'occasion d'un festin, voyant que tout le monde était de bonne humeur, il demanda la parole. Du mieux qu'il put, il leur dit qu'il les aimait, que pour eux il avait quitté son beau pays et ses frères bien-aimés.

C'est Celui qui a fait tout, dit-il, qui m'a donné cette affection envers vous; c'est lui qui a créé le premier homme, dont nous sommes tous issus. Voyant pourquoi n'ayant qu'un même père nous sommes tous frères et nous devons reconnaitre un meme Saigneur et Capitaine, nous devons tous croire en lui et accomplir ses volontés.

Saint Paul avait dit à peu près la même chose à Athènes devant l'Aréopage. Le Sorcier ici l'interrompit:

Quand je le verrai, dit-il, je croirai en lui. Autrement, non.

Lorsque tu me dis, réplique le Père, que ton père a tenu tel et tel discours, je te crois, me figurant qu'il n'est pas menteur. Tu crois bien qu'il y a un Manitou et tu ne l'as jamais vu.

Ton Dieu n'est pas venu en notre pays, repondit le Sorcier, voila pourquoi nous ne voulons pas croire en lui.

On se souvient que les Esquimaux de Mgr Turquetil lui avaient fait, eux aussi, la même objection. Et pour les Esquimaux un Jésus même né chez les Algonquins n'eut pas été plus des leurs qu'un Sauveur sorti du peuple juif.

Avec un argument à la portée de ses interlocuteurs, le Père Le Jeune leur rappelle qu'il n'y a pas seulement la connaissance visuelle, mais aussi la connaissance intellectuelle.

Quand tu passes devant une cabane délaissée et que tu vois des perches, des branches de pin, un feu qui fume encore et que tout ce que vous laissez lorsque vous décabanez, tu sais que des Sauvages sont passes par la... Ainsi, en voyant le soleil et les astres... 
Le Père demanda alors à Pierre Pastedechouan, l'apostat, de reprendre ses arguments et de les expliquer à son frère le Sorcier, comme il faut, dans sa langue. L'apostolat ne voulut pas ouvrir la bouche. Discuter religion dans sa propre langue le dépassait. Baptisé en France, c'est en français qu'il avait été catéchisé; en langue sauvage il ne peut parler que de choses matérielles. Le Père insista pour être interprété. Comme il s'enflammait quelque peu le Sorcier lui dit:

Nicanis, c'est-a-dire, ne te fache pas. Avec le temps tu parleras comme nous et tu nous enseigneras ce que tu voudras, toi, nous l'écouterons. Mais lui, (parlant de son frère), il n'a pas d'esprit, nous n'avons nulle créance en lui.

L'apostat refusa encore, peu après, ses services au Père, alors que celui-ci aurait voulu instruire de la religion une femme qui se mourait. Belle occasion pour le Père de faire un acte d'humilité. J'ai souvent souhaité, avoue-t-il, qu'un saint fût à ma place pour opérer en saint. Les petites âmes crient beaucoup et font peu; il faut se contenter de la bassesse.

Cette femme malade était l'épouse même du Sorcier. Le Père essayait de lui rendre service quand elle demandait quelque chose, afin de pouvoir lui dire quelques mots. Elle ne parlait pas quand les autres sauvages étaient auprès d'elle, et seule avec le Père elle répliquait les mêmes objections que son mari: Ton Dieu, on ne le voit pas. La mauvaise volonté de l'apostat fut que le Père ne put gagner davantage, à son propre témoignage.

Le Père Le Jeune aurait probablement eu le même résultat chez les Chinois. Chrétiens nous sommes faits à cette idée que le salut est affaire individuelle, personnelle, que sur ce point les femmes et les enfants ne dépendent pas totalement du mari ou des parents. D'après les coutumes païennes, la femme doit dire comme son mari, et même un beau-frère n'a pas à intervenir. L'apostat ne faisait que suivre le code des mœurs sauvages. Il aurait fallu que le Sorcier dise à sa femme d'écouter le Père, même si lui-même voulait rester païen. Mais il n'était pas assez avancé pour cela. 
Le 3 décembre, en la fête de saint François-Xavier, le Père Le Jeune voulut démontrer au Sorcier que tout le tintamarre qu'il faisait auprès des malades pour les guérir ne valait rien, et que prier Dieu serait bien plus efficace. Il lui lança même un défi, s'il voulait promettre d'en accepter sincèrement le résultat. Mais, il ne gagna pas grand'chose.

\section{NOËL. DANS LES BOIS}

Il aurait certes été intéressant de pouvoir établir de façon assez précise l'endroit où le Père Le Jeune a campé avec ses Montagnais, le 24 décembre 1633 . Nous avons cependant mieux que cela, puisqu'il nous a détaillé les tristesses et les joies de ce Noël-là.

Le 24 decembre, note-t-il, Notre-Seigneur nous donna un porc-épic gros comme un cochon de lait et un lièvre. C'etait peu pour 18 ou 20 personnes que nous étions. Mais la Sainte Vierge et son glorieux époux Saint Joseph ne furent pas si bien traités a meme jour dans l'étable de Bethleem.

Le lendemain, jour de réjouissance parmi les Chrétiens pour l'Enfant nouveau-né, fut pour nous un jour de jeûne; la faim qui fait sortir le loup du bois m'y fit entrer plus avant pour y chercher de petits bouts d'arbre que je mangeai avec délices. Des femmes ayant jeté aux chiens, par mégarde ou autrement, quelques rognures dont on fait des cordes de raquette, je les ramassai et fis un bon diner, quoique les chiens eux-meme quand ils auraient tant soit peu à manger, n'en voulussent pas gouter.

Ainsi préparée par un jeûne rigoureux, la fête de Noël, la première sur la rive droite du Saint-Laurent, eut tout de même sa solennité. Le Père continue:

Le même jour de Noël je m'en allai vers le soir visiter nos autres voisins. Il n'y avait plus que deux cabanes, Ekhennabanate avait pris un autre côte depuis 5 ou 6 jours, parce qu'il n'y avait pas assez de chasse pour tout le monde. Je les trouvai taciturnes.

L'apostat demanda quel jour il était. Il est aujourd'hui la fête de Noel, que je répondis. Se tournant 
vers le Sorcier il lui dit qu'a tel jour etait né le Fils de Dieu que nous adorons et appelons Jesus.

Le Pere lui dit: Dieu nous assistera si nous recourrons a lui.

Et il pria l'apostat de lui tourner en sa langue deux petites oraisons dont il dirait l'une et les sauvages l'autre. Sur l'heure le Père composa deux petites prières que l'apostat lui traduisit lui promettant en outre de lui servir d'interprète.

Le Père exultait. Il recommanda l'affaire à Notre-Seigneur et le lendemain matin il dressa un petit autel avec son crucifix et quelques images prises dans son bréviaire. Il appela alors les sauvages des deux cabanes et leur dit de son mieux en leur langue qu'il ne tiendrait à eux qu'ils fussent secourus. L'apostat, que la crainte de mourir faisait parler, approuva cette fois ces paroles, après que le Père eut lu ce qu'il avait sur son papier. Le Père dit aux sauvages:

Etes-vous contents de prier avec moi ?

Ils répondirent:

$\mathrm{Ni}$ mi roue ri te non, ni mi roue ri te nom,

Nous sommes contents.

Et tous répétèrent la prière avec lui.

Puis on se dispersa dans les bois environnants. Chacun prit quelque chose. Il n'y eut que l'apostat qui rentra les mains vides. Le soir Mestigoit apporta trois castors, reconnaissant le secours de Dieu dans la chasse qu'il venait de faire. L'apostat renchérit: Sans lui, nous n'aurions pas cela. Selon la coutume sauvage cela valait bien un festin.

Le Père pensa que l'occasion serait alors meilleure pour tirer une conclusion. Au temps voulu le Père Le Jeune voulut parler de reconnaissance envers Dieu pour cette chasse extraordinaire. Le Sorcier se mit en travers et la leçon, retardée de bonne foi, fut manquée. Tout n'était pas perdu cependant, car le Père eut dans la suite occasion de causer avec son hôte. Le Sorcier, d'ailleurs, quitta les autres le 13 mars. 


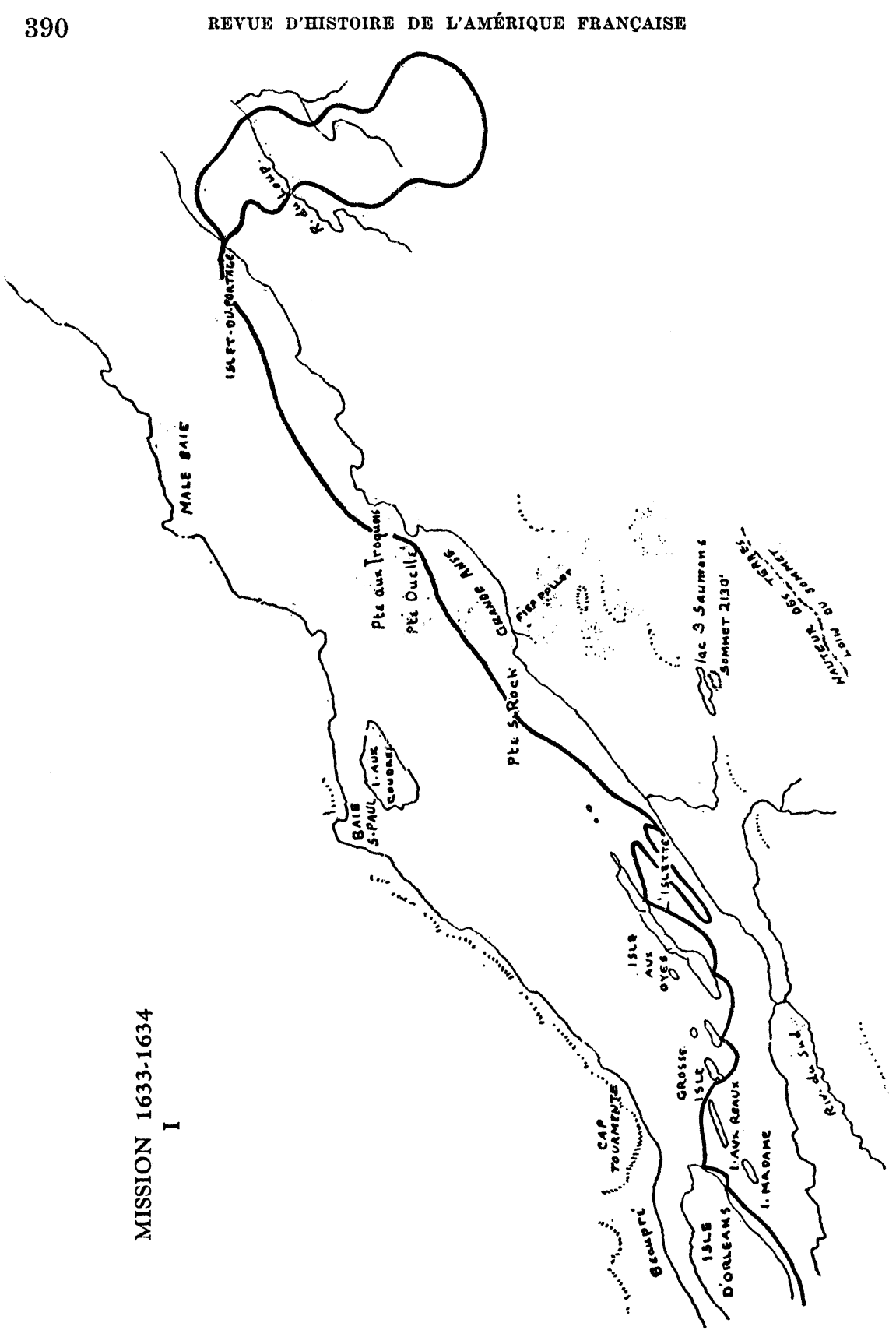


LA MISSION DU PÈRE PAUL LE JEUNE, S.J. SUR LA CÔTE-DU-SUD

391

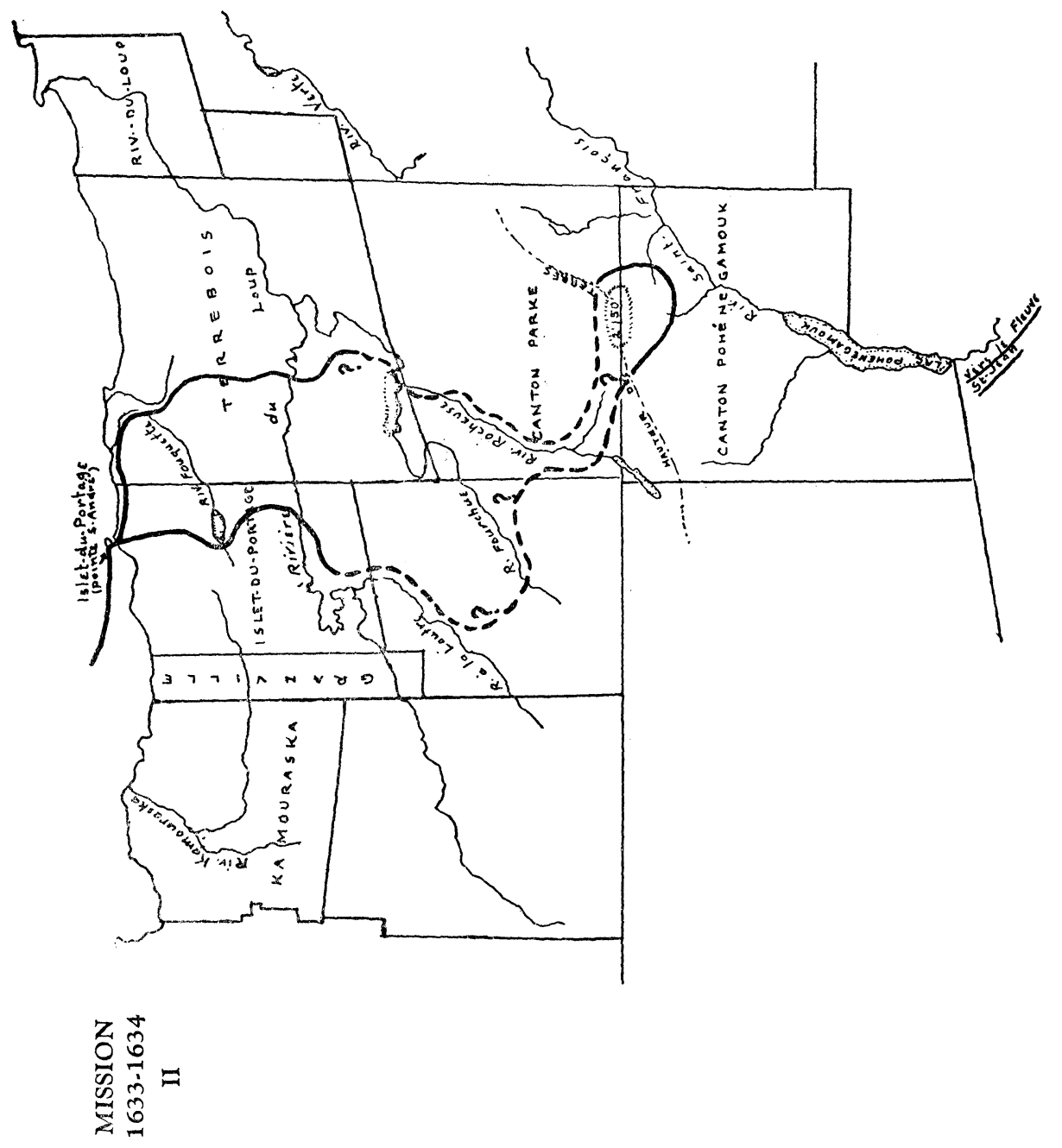


Plus à l'aise, Mestigoit prenait plaisir à demander au Père mille questions, sur le soleil, sur la terre et les antipodes, sur la France et son Roi Louis XIII.

- Tu me crois, lui dit le Père Le Jeune, quand je te parle des choses de la terre, pourquoi ne crois-tu pas de même quand je te parle des choses du ciel ? - Je te croirai, répondait le sauvage, quand tu sauras bien parler.

On peut bien énoncer en un langage rudimentaire les principales vérités. Mais pour comprendre les objections qui peuvent surgir dans les esprits, pour faire les distinctions nécessaires à une bonne réponse, il faut avoir plus qu'un vocabulaire enfantin ou commercial. Le Père Le Jeune le savait plus que tout autre.

Mais ses hôtes ne pouvaient soupçonner l'héroïsme que demandait à un Français un hivernement entier au milieu d'eux.

\section{Y EUT-IL VRAIMENT MISSION?}

Mission dit aussi : fondation, continuation.

La tournée de 1633-1634 du Père Le Jeune n'a ressemblé en rien à ces prises de possession territoriale par la plantation d'une croix aux armoiries du Roy de France. Sa prise de possession est toute spirituelle: apprendre la langue pour atteindre les âmes. Aucune marque sur le terrain pour nous dire qu'il est passé par là. Pas même une croix. Il se passera sept ans encore avant qu'une croix soit érigée sur la Côte-du-Sud, à la Pointe-à-Lacaille, en souvenir d'un noyé.

La randonnée du Père Le Jeune contribue cependant à l'avancement des missions de la Nouvelle-France en ceci qu'il a conclu qu'il était impossible de christianiser des peuplades ne vivant que de chasse, sans les amener à une vie plus sédentaire. D'où, trois ans après, l'on verra surgir la bourgade de Sillery près Québec. Le Père Le Jeune en sera le supérieur de 1639 à 1641.

Si, sur la Côte-du-Sud, dans les endroits visités par le Père Le Jeune, il n'y a pas eu de mission d'établie, c'est que les Sau- 
vages n'y étaient pas en assez grand nombre, et en passant seulement.

L'abbé Alexandre Paradis a relevé dans les registres de Kamouraska, en juin 1756, la sépulture du Père Quoad, jésuite, missionnaire des sauvages de la rivière des Caps. L'on ne trouve pas le nom de ce père dans la liste des Jésuites venus en Canada.

Il y aura toutefois sur la Côte-du-Sud des sauvages devenus chrétiens, on ne sait comment. Ainsi, en 1715, Blondeau, seigneur de la Rivière-du-Loup, prend comme parrain d'un de ses fils un chef Malécite de la Rivière-Trois-Pistoles, avec une marraine de la même tribu. De même, lorsque l'église de la Grande-Anse, incendiée en octobre 1766, est réparée l'année suivante, une souscription de 18 livres faite par les sauvages est mentionnée par N.-E. Dionne.

$\mathrm{Au}$ dire de l'abbé Raymond Casgrain, Mgr de Saint-Vallier a songé pendant quelque temps à établir à la Rivière-Ouelle une mission pour les sauvages; c'était un des endroits qu'ils fréquentaient volontiers.

Beaucoup plus tard, en 1798, Mgr Plessis autorise le curé Perras, de Saint-Jean-Port-Joli, à desservir les nomades qu'il a dans sa paroisse; M. Gérard Ouellet voit là une justification de l'appellation "Anse aux Sauvages" donnée à l'anse à Éloi Caron. Nomades, ils l'étaient certes. Ceux de l'Anse aux Sauvages à Saint-Jean, du ruisseau des Prairies à Sainte-Louise, pouvaient bien être de ceux qui campaient au Cap-Martin, et dont Mme Louis Ouellet de Saint-Roch, née en 1832, avait gardé le souvenir.

Dans les registres de quelques paroisses l'on trouve le nom de certains sauvages chrétiens. Mgr Tanguay en relève 8 à SaintThomas, 3 au Cap-Saint-Ignace, 13 à la Rivière-Ouelle. Il s'agit d'ordinaire de tout jeunes enfants, sans noms de famille, comme si ces baptisés étaient des enfants achetés et élevés par des familles canadiennes, comme dans le cas du capitaine Côté de Saint-Thomas qui est nommé.

Sainte-Anne-de-la-Pocatière a aussi quelques actes de sauvages. L'on ne trouve nulle part de nom de famille héréditaire 


\begin{tabular}{|c|c|c|c|}
\hline $\begin{array}{l}\text { MARIA GES } \\
\text { CELÉBRES EN LEGLISE } \\
\text { VOTIVE DE } \\
\text { NOTRE-DAME } \\
\text { DE } \\
\text { RECOUVRANCE } \\
\text { DEPUIS SA CONSTRUCTION } \\
\text { EN } 1633 \\
\text { JUSQU'A LINCENDIE OU } \\
14 \text { JUIN } 1640\end{array}$ & $\begin{array}{l}\text { PRIA GES } \\
\text { ÉBRES EN LEGLISE } \\
\text { VOTIVE DE } \\
\text { TRE-DAME } \\
\text { DE } \\
\text { COUVRANCE } \\
\text { IS SA CONSTRUCTION } \\
\text { EN } 1633 \\
\text { QU'A LIINCENDIE OU } \\
4 \text { JUIN } 1640\end{array}$ & & $\begin{array}{c}\text { MARRIAGES } \\
\text { CELLBRATE IN THE } \\
\text { VOTIVE CHURCH OF } \\
\text { NOTRE DAME } \\
\text { DE RECOUVRANCE } \\
1633-1640 . \\
\text { THE ANCESTORS OF ALMOST } \\
\text { ALL FRENCH CANADIAN } \\
\text { FAMILIES IN CANADA AND } \\
\text { EASTERN UNITED STATES }\end{array}$ \\
\hline \multicolumn{2}{|c|}{ ATE EPOUX - SPOUSE } & \multicolumn{2}{|c|}{ OFFICIANT TEMOINS - WITNESSES } \\
\hline & Nó́l Langl OIS-FrançOISE GRENIER & \multicolumn{2}{|c|}{\begin{tabular}{|l|l} 
C. LÉsUITES) & ROBERT GIFFARD \\
NOËL JUCHEREAU
\end{tabular}} \\
\hline 634 & GUILL HÉBERT-HÉLĖNE DESPORTES & C.Lalemant & $\begin{array}{l}\text { HENRY PIR } \\
\text { ROBERT G }\end{array}$ \\
\hline $\begin{array}{l}1635 \\
1 \times-9\end{array}$ & JEAN BOURDON-CATHERINE Potel & P. Le deune & JUC \\
\hline $\begin{array}{r}1635 \\
\times 1-17 \\
\end{array}$ & JEAN CÔTÉ-ANNE MARTIN & C. Lalemant & \\
\hline $\begin{array}{r}1035 \\
\times 1-20 \\
\end{array}$ & Martin Grouvel-Marg. Auber & C. Lalemant & 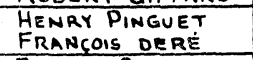 \\
\hline $\begin{array}{r}1636 \\
\times 1.30 \\
\end{array}$ & Jamen Bourguignon-Claire Morin & hicolas Odam & $\begin{array}{ll}T C \\
T C \\
T S\end{array}$ \\
\hline $\begin{array}{r}0.78 \\
1637 \\
1-14\end{array}$ & ANTOINE BRASSARD-FRANCSOISE MÉRY & C.Laleniant & 016 \\
\hline \multirow{2}{*}{$\begin{array}{c}1637 \\
v 11-12 \\
\end{array}$} & ROBERT DROUIN-ANNE CLOUTIER & \multirow[t]{2}{*}{ S.Lalemant } & \multirow{2}{*}{$\begin{array}{l}\text { OLIVIER LE TARDIF } \\
\text { FRANĢOIS DEREE }\end{array}$} \\
\hline & FRANCOIS BÉLANGER- MARIE GUYON & & \\
\hline \begin{tabular}{|l|l|}
1637 \\
$1 x-21$ \\
\end{tabular} & Jacques Selle- Marie Béraro & Jean Dequen & \\
\hline $\begin{array}{r}1637 \\
\times-7 \\
\end{array}$ & Jean Nicolet-Marg.Couillard & Cl.Pyart & \\
\hline $\begin{array}{r}1637 \\
\times-25 \\
\end{array}$ & Robert Caron-Marie CREVET & C. Lalemant & Amen Ba \\
\hline $\begin{array}{r}1637 \\
\times 1.3 \\
\end{array}$ & OLIVIER LE TARDIF- LSE COUILLARD & P. Le Jeune & \\
\hline $\begin{aligned} 1638 \\
V-13 \\
\end{aligned}$ & Denis Du Guar-Catherine Gautier & C.Quantion & \\
\hline $\begin{aligned} 1638 \\
-22 \\
\end{aligned}$ & ETIENNE Racine-Marg. Martin & hes adam & \\
\hline & FRANCOIS DROUET-PERRINE GODIN & Q Quantin & \\
\hline $\begin{array}{l}1639 \\
\text { Vil1.-8 } \\
\end{array}$ & LAvde Poulin-Jeanne Merc & & ROEERT \\
\hline $\begin{array}{r}1639 \\
1 \times-3 \\
\end{array}$ & GUILL.BIG OT - MARIE PANIS & hus. & \\
\hline $\begin{array}{l}1639 \\
1 \times-12 \\
\end{array}$ & Jean Gouray- Isabeau Panis & his. & $\begin{array}{l}\text { NICOLAST } \\
\text { JEAN LÉGE }\end{array}$ \\
\hline $\begin{array}{l}1639 \\
1 \times-26 \\
\end{array}$ & Jacques Maheu-Anne $c c$ & his. & 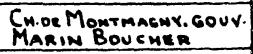 \\
\hline & JEAN JOLLIET-MARIE D'ABAN COUR & his.a & \\
\hline & NOËL MORIN-HELÉne DEsportes, veuve & hic I Idam & $\begin{array}{l}\text { NiCOLAS P } \\
\text { RODERT G }\end{array}$ \\
\hline
\end{tabular}

Original encadré donné à $M$. Fred Wong, propriétaire du Wong's Cafe, 19 rue Buade, sur le présumé site de Notre-Dame-de-Recouvrance. 
chez ces peuples; aussi le plus simple était d'écrire, comme on voit en 1830: Pierre Sauvage. Autour de 1900 il y a encore à Sainte-Louise une veuve Pierre Sauvage, qui "travaille en journees, et a son banc à l'église".

Si les sauvages n'ont pas été assez nombreux pour une mission, par contre les missionnaires, Récollets et Jésuites, ont dû, à partir de 1680 environ, s'occuper des colons français. La plus ancienne paroisse, peut-être, de la Côte-du-Sud, le Cap-SaintIgnace, rappelle le travail des Pères de la Compagnie de Jésus.

C'est vers cette époque que la première messe sur la Côtedu-Sud eut lieu. Le Père Le Jeune ne l'a pas dite, au cours de son voyage; il devait avoir sa chapelle, puisqu'il s'était apporté du vin ... Vin que l'apostat a bu dans une des îles... .

Le Père Le Jeune a cependant consacré son itinéraire par la récitation, et combien pénible, de son bréviaire. Il écrivait:

J'ai cru plus d'une fois que j'allais etre aveugle (a cause de la fumée), les yeux me cuisaient comme feu, ils me pleuraient ou distillaient comme un alambic; je ne voyais plus rien que confusement a la facon de ce bon homme qui disait: Video homines velut homines ambulantes.

Je disais les psaumes de mon breviaire comme je le pouvais, les sachant a demi par coeur, j'attendais que la douleur me donna un peu de relache pour reciter les lecons et je venais a les lires. Elles me semblaient ecrites enlettres de feu ou d'ecarlate. J'ai souvent ferme mon livre, n'y voyant que confusion qui me blessait la vue.

Continuation missionnaire, certes, par la Communion des Saints. Mais autre est celui qui sème, autre celui qui moissonne.

ADRIEN CARON, ptre 